\title{
What do we know about the impacts of the Marine Stewardship Council seafood ecolabelling program? A systematic map protocol
}

\author{
Ashleigh Arton ${ }^{1 *}$, Anthony Leiman² ${ }^{2}$ Gillian Petrokofsky ${ }^{3}$, Hilde Toonen ${ }^{4}$, Francis Neat ${ }^{1}$ and Catherine S. Longo ${ }^{1}$
}

\begin{abstract}
Background: Voluntary sustainability standards and eco-labels are market-based mechanisms used to encourage producers and consumers toward environmental sustainability. The Marine Stewardship Council (MSC) is one such program which aims to improve the state of the world's oceans and promote a sustainable seafood market. Now in its 21 st year, with approximately $14 \%$ of global fisheries landings certified, there is growing evidence of the program's impacts (direct and indirect) on factors from fisheries management and consumer awareness to coastal communities' livelihoods and international law. To better understand the program as a whole, the proposed systematic map will collate and describe published research on the environmental, social and economic effects of the MSC program, and indicate the prevalence of disciplines or topics and study designs in this literature. Areas considered of greater interest, knowledge gaps, and future research priorities will be identified.
\end{abstract}

Methods: This systematic map protocol describes how research regarding the MSC will be searched, identified and described. All research on the MSC and its effects (direct and indirect) will be included. The review is not limited to effects on certified fisheries but will include those on supply chain companies, socio-economics of coastal communities, governments, biological populations and ecosystems, NGOs and other stakeholders impacted by or potentially influenced by the MSC. The search scope includes studies from MSC's foundation in 1997 to the present. To identify studies, pre-determined inclusion and exclusion criteria will be used at the title, abstract and full text levels. In addition to the use of bibliographic databases and internet search engines, the authors will call for and search for grey-literature. The final systematic map will be presented in a descriptive report detailing the focus, extent, and occurrence of research on the MSC's impacts, taking special care to map the disciplines focused on the programme and the study design of research.

Keywords: Voluntary sustainability standard, Sustainable fisheries, Market-based mechanisms, Impact evaluation

\section{Background}

The Marine Stewardship Council (MSC) is an international voluntary sustainability standard-setter and seafood ecolabelling program. Established in 1997, the MSC's mission is to contribute to the health of the world's oceans by promoting sustainable fisheries and effective management through its third-party certification

\footnotetext{
${ }^{*}$ Correspondence: ashleigh.arton@msc.org

${ }^{1}$ Science and Standards, Marine Stewardship Council, London, UK

Full list of author information is available at the end of the article
}

scheme [1]. By utilizing its ecolabel as a market-based incentive and rewarding sustainability, the MSC encourages improvements 'on the water', by certifying fisheries that have demonstrated good environmental practices as articulated in the MSC's Fishery Sustainability Standard [2]. For a product to exhibit the MSC ecolabel, all companies in the supply chain that handled that product must be certified under the MSC Chain of Custody $(\mathrm{CoC})$ Standard [2] which guarantees full traceability of the product from a certified sustainable source. 
The MSC Fisheries Standard defines fishery sustainability on the basis of the guidelines of Food and Agriculture Organisation of the United Nations (FAO) code of conduct for responsible fisheries [3], and strives to incorporate internationally-established best practice and best available science. The Fishery Standard requirements focus on three principles: healthy target stock status; mitigation of environmental impacts; and effective management [4]. In order to achieve and/or maintain certification, fisheries might need to, among other things, reduce fishing effort, establish new harvest strategies, mitigate fishing impacts on vulnerable species, habitats and ecosystems, change governance or policy practices, or define fishery-specific management.

MSC's mission as an organisation is underpinned by its theory of change (ToC), see Fig. 1. A ToC describes how or why a change or desired outcome is hypothesised to occur within a given context. The MSC's ToC suggests that recognising sustainable fishing practices with certificates and eco-labels creates market incentives to improve fishing practices. When a consumer chooses to purchase MSC certified seafood over another seafood product, certified fisheries are rewarded for their sustainable practice through that market preference. Furthermore, purchasing preferences may go beyond individual consumers to include processors, suppliers and retailers who can make commitments to sourcing MSC certified products. Such preferences may increase the global demand and market access for certified fisheries and provide the incentives needed for fisheries to undergo the rigorous assessments to potentially become MSC certified. Increasing numbers of certified fisheries, coupled with greater consumer awareness may incentivise further fisheries that are operating below the MSC standard to make sustainable changes 'on the water' in line with the organisation's principles. For example, to align with principle 2 of the Fishery Standard (minimising environmental impact) a fishery may adjust its practices to ensure that other species and habitats in the ecosystem remain healthy. This could mean fishing at night to avoid accidental bird entanglements or collecting information on seabed impacts so as to better manage the potential effects of fishing activities.

In order to qualify for MSC certification a minimum score of 60 must be achieved for each of the 31 principle issues in the Standard and fisheries must achieve an average score of 80 overall for each of the three Principles (determined by the average of the principle issue scores under that Principle) [5]. Where a fishery scores above 60 but below 80, a fishery is given a 'condition' where that score must be improved to the 80 level over the certification period (usually 5 years) [5]. The MSC allows fisheries to qualify for MSC certification without meeting the 80 level on all indicators because the movement of fisheries from the 60 to 80 levels is where fisheries are able to transparently improve their practice and undergo surveillance in this period. If a fishery fails to a 'close' a 'condition' within a set time period, their certification will be taken away. This is one of the tenets of MSC's theory of change as it contributes to improved fisheries practice. This process is broadly outlined in Fig. 1 .

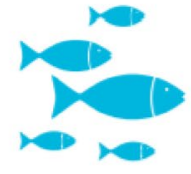
the MSC Standard are independently certified as sustainable

\section{Consumers preferentially purchase seafood with the MSC ecolabel}

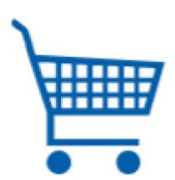

Retailers and restaurants choose MSC certified sustainable seafood
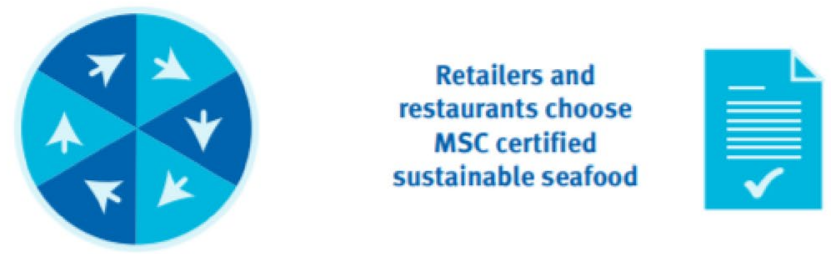

A traceable supply chain assures consumers that only seafood from an MSC certified fishery is sold with the MSC ecolabel

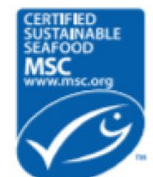

Fig. 1 The Marine Stewardship Council's theory of change describes how the organisation envisages itself contributing to more sustainable seafood practices 
This process can result in environmental impacts (changes in the health of target fish stocks or more effectives fisheries management) as well as impacts on the market (greater demand and/or supply of sustainable seafood or consumers willing to pay price premiums for ecolabelled products). Importantly the changes that occur due to fisheries voluntarily complying with the MSC's standards or through the 'closing' of 'conditions' directly align with the principles and criteria in the standards themselves. For example, Principle 1 states that: "The fishing activity must be at a level which is sustainable for the fish population. Any certified fishery must operate so that fishing can continue indefinitely and is not overexploiting the resources" [4]. Here, to attain a score of 80 (for one of the principle issues within Principle 1) there "shall be evidence that the stock is at the target reference point now or has fluctuated around the target reference point for the past few years" [4]. As such the term 'sustainable' is linked to the target reference point of a stock (i.e. the amount of biomass of a particular stock believed to allow the population to maintain a sustainable yield). Thus, the MSC can only claim to create changes in line with this definition of 'sustainable'. In other words, the ToC does not just outline potential sustainable changes on the water but specific changes that directly align with the principles and criteria in the MSC's Standards.

The potential changes described in the $\mathrm{ToC}$ and their outcomes have been described in published literature. These include effects of MSC certification on fisheries management $[6,7]$, supply chains $[7,8]$ and the eco-labels impact on consumer awareness $[9,10]$. There are further published reported impacts that are not described in Fig. 1. These include effects on local economies [11], coastal communities [8], and international law [12]. It is this diverse range of impacts that this systematic map aims to capture. To this end, a comprehensive catalogue of relevant studies across disciplines, methods and geographic regions will provide an overview of knowledge clusters and knowledge gaps. Such an overview will contribute to elucidate the state of knowledge on how fisheries sustainability standards perform and operate, and answer Cooke et al. "Call for evidence-based conservation and management of fisheries and aquatic resources" [13]. This is important given that a search of articles published by Environmental Evidence, the official journal of the Collaboration of Environmental Evidence, finds few systematic maps and reviews that deal explicitly with marine fisheries except Araujo et al. [14], Hughes et al. [15] and Leisher et al. [16] and none that focus on certification and/or eco-labels.

While literature regarding the MSC has been included in previous systematic mapping and review exercises [17-19], these studies have focused exclusively on final outcomes of certification and sustainability standards (e.g. practice adoption or environmental impacts). Here, we seek a comprehensive overview of all the aspects of the MSC program that researchers have focused on, including management and policy processes, consumer awareness and willingness to pay, to name a few.

Systematic reviews of different sustainability standards rely on evidence typology and inclusion criteria [e.g., PICO (population, intervention, comparator, outcomes) or study design such as BACI (Before, after, control, intervention)] that may be difficult to apply to studies of changes in fisheries management [20]. For example, wild capture fisheries occur in highly dynamic environments, with harvesters and marine populations shifting spatially and temporally. These are harder to monitor than crops or forests that are spatially fixed and clearly delimited entities. In addition, potentially broad spatial scales over which some species are distributed, (such as the wide migratory range of tuna populations), or the vertical distribution of vulnerable ecosystems in the water column, make it costly and difficult to conduct randomised control trials or collecting information on covariates that control for such effects. This means that very strict selection filters risk excluding a large proportion of studies and, with them, potentially valuable insights. To avoid this, the PICO framework will be applied. Studies that lack comparators will still be included but will be noted separately. Also, any study design will be included.

Herein summary, we propose to map all the literature on the MSC without narrowing the scope based on study design. To our knowledge such a broad yet systematic review exercise has not been undertaken before. This document has been developed following the RepOrting standards for Systematic Evidence Syntheses (ROSES) for systematic map protocols [21] and the CEE Guidelines and Standards for Evidence Synthesis [22].

\section{Stakeholder engagement}

There are various definitions of stakeholder in the literature. According to the 'ROSES for systematic map protocols' a definition of stakeholder should be used to include researchers and decision-makers with a stake in the systematic map being created. As seen in Table 1 of Haddaway et al. [23] the term can refer to "People that have an interest in the subject matter: includes researchers and experts", "someone who has a stake in the findings" or "those ...that use the information from a systematic review". Haddaway et al. [23] also encourages a broader inclusion of stakeholders potentially affected by the results of the map. As such, the following methods and questions have been formulated and developed with input from MSC staff and external researchers who have expertise in fisheries biological sciences, marine 
ecology, the development and application of the MSC's standards, social science, and economics. The stakeholders were chosen for their expertise, covering the array of disciplines that tend to focus on the MSC, thus ensuring that all aspects and perspectives of the programme will be captured in the mapping process, as well as taking diverse perspectives into account. The MSC staff have a stake in the findings, are most likely to be affected by the review and have decision-making capabilities. The external researchers are interested in the subject matter and may use the information from the review. While this is not an exhaustive representation of all stakeholders potentially affected by the results of the review, these stakeholders will nevertheless "ensure that inputs and outputs are of the greatest relevance and reliability to all interested parties" [23]. The stakeholder described will provide input throughout the review process including the coding, analysis and write-up stages. Meetings with stakeholders were held at the MSC headquarters office in London on 25 April 2018 and remotely on 30 April 2018 to formulate the research questions, search techniques, inclusion and exclusion criteria and discuss the available evidence in general.

\section{Objective of the review}

This proposed systematic map aims to identify evidence of the impacts of MSC and to collate and describe the topic areas of research undertaken to date on the environmental, social and economic effects of the MSC program. It will also collect and describe the type of evidence thee articles draw on. This will help identify areas most commonly researched, trends in the research over time, and knowledge and data gaps, and will help identify potential future research priorities for external researchers as well as MSC's Monitoring and Evaluation program.

\section{Primary question}

What is the evidence for impacts of the MSC program?

This question has the following components:

- Population Any harvester groups or chain of custody companies, governments, communities, biological populations and ecosystems potentially impacted by MSC certification or have the potential to become MSC certified and thereby potentially impacted by such certification.

- Intervention MSC certification (according to the MSC Fisheries or Chain of Custody standards).

- Comparator Absence of intervention either between fisheries, countries, sites or groups, and/or over time.

- Outcome Positive, negative, neutral or ambivalent impacts on ecosystems, trade, socio-economic outcomes, governance, management or policy.

\section{Secondary questions}

What are the characteristics of documented evidence in terms of geographic location, and focus of the study (e.g. consumer willingness to pay, program legitimacy and credibility, fisheries bycatch etc).

- What are the characteristics of the fisheries that are most reported in the literature?

- What are the primary disciplines that focus on the MSC programme and its effects?

- What study designs are used in research regarding the MSC programme?

- What are the types of outcomes (e.g. socio-economic, ecological, policy recommendations etc) for which evidence is documented?

- What is the frequency of these documented outcomes?

- What are the current knowledge clusters? To be identified by examining the characteristics of the fisheries, disciplines and study designs.

- Where do gaps exist in the evidence base that may be prioritised for future research?

\section{Methods \\ Searching for articles}

The articles to be screened for the systematic map will be found using the following Boolean string:

"Marine Stewardship Council" OR "fisheries certif"" OR "certif" fisheries" OR "seafood eco-label" OR "seafood ecolabel" OR "seafood eco label".

This search string is applicable to all three of the databases that will be searched (see below). No restrictions on document type will be applied, however only literature published between 1997 and the present will be included as 1997 is the year of MSC's founding. Note that the first fisheries were certified in 2000 .

All database, search engine and grey literature searches will take place in English.

Searches in the publication databases will be performed using university library subscriptions from universityaffiliated researchers on our team (FN, AL, HT and GP). Where an article is unavailable using one institution's subscriptions, access to it will be sought elsewhere by another team member.

\section{Estimating the comprehensiveness of the search}

The comprehensiveness of the search strategy will be assessed by comparing results to a list of benchmark articles. This test-list of articles is the same used for the scoping exercise (see Additional file 1: Scoping). The ten papers in the list were deemed by the advisory group as paragon examples of articles that are relevant to the 
primary and secondary questions. The list covers a range of authors and topics including articles on environmental impacts, management, governance, consumer awareness and the developing world, including quantitative analyses of stock assessments to more qualitative understandings of stakeholder perceptions of the program.

\section{Publication data bases}

The following online databases will be searched:

1. Clarivate Analytics Web of Science ${ }^{\mathrm{TM}}$ Core Collection http://apps.webofknowledge.com/.

2. Elsevier's SCOPUS http://www.elsevier.com/onlin e-tools/scopus.

3. AGRIS database http://agris.fao.org/agris-search/ index.do.

\section{Supplementary searches}

In addition to searches in databases, hand searching in MSC's own grey literature and other organisational websites will be done (including WWF, FAO and ISEAL Alliance). Literature will also be provided directly by members of the advisory group and call for extra literature through their own contacts and other related stakeholders. The references from other relevant reviews [17-19] will be also be included if they have not already been found though the above searching.

\section{Search engines}

The same search terms will also be applied in Microsoft Academic (http://academic.research.microsoft.com) and Google Scholar (https://scholar.google.co.uk) in order to source additional grey literature not found elsewhere [24]. For these searches, 'Harzing's publish or perish' plug-in will be used to extract the first 250 entries, following the recommendation by Haddaway et al. [24]. Publish or Perish is a software program that retrieves and analyses academic citations. It uses a variety of data sources (incl. Google Scholar) to obtain raw citations. Harzing's Publish or Perish is available at: https://harzi ng.com/resources/publish-or-perish. Articles found with Publish or Perish will be screened in a similar fashion to the databases. Changes in the above search string will be formatted to be compatible with the search engines.

\section{Article screening and study eligibility criteria Screening process}

Following the completion of all searches, these will be imported into Colandr (a product of a collaborative partnership between the Science for Nature and People Partnership Evidence-Based Conservation working group, DataKind, and Conservation International-https ://www.colandrapp.com/) to allow screening by multiple screeners. Colandr provides a platform for all systematic mapping requirements, from title and abstract screening to meta-data extraction, and applies machine learning and natural language processing algorithms to sort articles according to relevance which can reduce the time taken to screen compared with traditional methods [25].

An exercise to measure inter-rater agreement using Cohen's kappa [26] will be undertaken before title, abstract and full-text screening and meta-data extraction [27]. A random sample of $10 \%$ of the literature will be screened by the reviewers according to the inclusion and exclusion criteria detailed below and their results directly compared. Where conflicts exist, they will be discussed and rectified to ensure all reviewers agree. Once a Kappa score of $\geq 0.6$ has been achieved, individual screening will commence.

In order to ensure consistency of decisions, minimum of $10 \%$ of articles will be reviewed by two coders at the title, abstract and full text screening levels. Where disagreements occur, the coders will discuss them and come to a consensus to either include or exclude articles. As this iteratively occurs through the different screening levels, the coders' decision-making and reasoning processes are likely to converge to increase the level of consistency. All screening (at title, abstract and full-text levels) will be performed by 2 non-MSC coders.

It is recognised that the MSC, on occasion, plays a significant role in individual fisheries management and policy debates. For example, MSC representatives may be present at annual stock assessment meetings and discussions may be framed around MSC relevant issues. This may be captured in the meeting minutes. It is acknowledged while these minutes and similar documents may be an important resource and provide a holistic understanding of the MSC and its impacts, they cannot be included in this systematic map due to concerns regarding sampling bias, veracity, transparency and repeatability.

All included will be recorded and included in the final report. A list of articles excluded at full text with reasons will also be available.

\section{Demonstrating procedural independence}

Readers that have authored articles that are identified in the search process will not be able to read these or comment on their inclusion or exclusion. When this occurs, the author will refer the article to another reviewer.

\section{Eligibility criteria Eligible populations or subjects}

Relevant subjects are fisheries, companies, governments, communities and biological populations or 
ecosystems that have been or are potentially being certified or involved in the certification process. Additionally, those fisheries, companies, governments, communities and biological populations or ecosystems potentially affected through certification of other subjects are also eligible populations.

\section{Eligible intervention}

The eligible intervention in question is the MSC programme. This can include a certified fishery or chain of custody actors and any subjects potentially becoming certified according to the fishery or chain of custody standard (although these will be noted separately).

\section{Eligible comparators}

The absence of MSC certification (or potential MSC certification) either between fisheries, governments, sites or groups, and/or over time will be included. Articles that do not have a comparator will be included but noted separately. This will be done to include social science and political science studies and others that may lack a comparator, but whose outcomes are still of importance to this study.

\section{Eligible outcomes}

Any outcome related impacts on ecosystems or biodiversity, trade, socio-economic outcomes, governance, management or policy will be included. Examples may include: the effects of MSC certification on stock health, fishing mortality or gear use; the effects of the MSC certification process on interactions between stakeholders (i.e. changes in partnerships or conflict) or changes in consumer awareness regarding environmental sustainability in the marine realm or changes in consumers' willingness to pay for MSC eco-labelled products.

\section{Eligible types of study design}

All types of study design will be included with the study type recorded. Only literature that does not pass the above PICO specifications, language and time limitations will be excluded. This will be done to get an understanding of the evidence generated about the MSC.

\section{Study validity assessment}

No formal, systematic study validity assessment of the included studies will be undertaken. This is not mandatory for systematic maps [28]. However, metadata pertaining to study design and data collection methods will be extracted.

\section{Data coding strategy}

Following full text screening, meta-data will be extracted from the included studies. All data extraction will be performed in Colandr using a data-extraction form developed in the online platform according to the following main categories and labels:

1. Bibliographic information (title, authors, date, journal, year).

2. Literature type (grey literature, journal article, conference proceedings etc).

3. Discipline (political science, economics, fisheries science etc).

4. Geographic information (country, continent, coordinates, development level based on the world bank list of economies).

5. Type of study design, data collection methods and quantitative/qualitative.

6. Fishery information (if particular fishery/fisheries are identified. E.g. certified fishery name, species, gear, sea, FAO major fishing area, fisheries scale).

7. Study focus (e.g. fish stocks, habitats and ecosystems, transparency in the supply chain etc).

8. Intervention type (certified fishery, certified $\mathrm{CoC}$ actor, prospective certified entities, fishery or $\mathrm{CoC}$ actors in assessment).

9. Outcome (as described in the study).

10. MSC impact (presence of MSC impact, description of specific MSC impact, whether the impact is intended/unintended according to the MSC's standards and whether the evidence for the impact is direct or indirect (proxy/circumstantial).

11. MSC impact tested against the MSC's intent (whether the study examines MSC's impact or credibility against MSC's own Theory of Change and/or vision and mission, and/or standards. I.e. the study does not test the MSC's impacts against aspects outside of the program's remits).

For a detailed overview of the labels, descriptions, input methods and rationales for the data-extraction form (including screen shots of the Colandr form) see Additional file 2: Data extraction sheet. This extraction form has been piloted using the initial scoping exercise test searches performed using web of science $(01 / 06 / 2018)$ that recovered 162 articles. For the pilot, 10 articles were coded by 2 non-MSC and 2 MSC staff using a pilot data extraction sheet (see Additional file 3: Pilot data extraction sheet). The pilot testing allowed the authors to refine, streamline and reduce ambiguity in the final data-extraction sheet.

Before full data coding commences, an exercise to measure inter-rater agreement using Cohen's kappa [26] 
using a random sample of $10 \%$ of the included articles will be performed. During this exercise any inconsistencies will be discussed between all coders and agreements made by consensus. The exercise will be repeated until a Kappa score of $\geq 0.6$ has been achieved, after which individual coding will take place. This will not only ensure inter-rater agreement but also the consistency and repeatability of the study.

During individual coding where data is missing, unclear or ambiguous, the review team will search for additional information and make changes according to consensus. Where data is taken from elsewhere references to the extra sources will be cited to ensure repeatability. Additionally, any uncertainties and issues that arise during the data extraction process will be flagged by the coder. Flagged issues will be dealt with in semi-regular meetings and decisions to rectify the problem made unanimously. This will further ensure consistency in coding. To help with repeatability these decisions will be documented and made available in an additional file in the final systematic map report. Coders that have authored included papers will not code these articles. Data extraction will be performed by 2 non-MSC coders and 2 MSC staff. To reduce the risk of partiality the majority (up to $75 \%$ ) of meta-data extraction will be performed by non-MSC staff.

The final extracted meta-data will be made included with the final report as additional files.

\section{Study mapping and presentation}

The final map and narrative will be described in accordance with the primary and secondary questions (see above). Further discussion will explain the relevance and highlight the knowledge gaps, clusters and areas for future research and their relation to the MSC's monitoring and evaluation programme. To identify knowledge gaps and clusters cross tabulations of key variables will be used to create heatmaps and highlight areas with high and low concentrations of studies. The advisory group plans to meet once the results have been collected to set arbitrary but specific cut off points which could then be used to identify study areas or topics that lack evidence and are poorly studied areas. The same will be done to identify knowledge clusters; topics or study areas deemed to have sufficient studies to allow for meaningful synthesis. Figures describing the outcomes for each of the secondary questions will also be included (e.g. a bar graph showing the number of studies per country or number of studies per certified species). This will all be presented in a systematic map that will include this as well as the full methodology undertaken and links (via the additional supporting files) to a full database of all included studies with their meta-data.

\section{Additional files}

Additional file 1. Scoping

Additional file 2. Data extraction Sheet.

Additional file 3. Pilot extraction sheet.

\section{Authors' contributions}

All the authors contributed to the formulation of the research questions and methodology. KL and AA conceived of the study and participated in its design and coordination. AA was a major contributor to the writing of the manuscript. KL, TL, GP, FN and HT helped in the drafting of and review of the study. All authors read and approved the final manuscript.

\section{Author details}

${ }^{1}$ Science and Standards, Marine Stewardship Council, London, UK. ${ }^{2}$ School of Economics, University of Cape Town, Cape Town, South Africa. ${ }^{3}$ Department of Zoology, Long-term Ecology and Resource Stewardship Laboratory, Biodiversity Institute, University of Oxford, Oxford, UK. ${ }^{4}$ Environmental Policy Group, Wageningen University and Research Centre, Wageningen, The Netherlands.

\section{Acknowledgements}

We thank Ellen Baker for her help and insight into systematic maps methodology.

\section{Competing interests}

$A A, F N$ and $C L$ are employees of the MSC. To avoid this being a potential conflict of interest, the following precautions were taken: non-MSC authors have been involved in the development of the manuscript and checked for the risk of selective or biased questions and methodology. The non-MSC authors have different backgrounds and expertise to ensure a diversity of stakeholder perspectives. The formulation of all questions and methods was done with the input of all authors. The CEE guidelines and ROSES form were closely followed to ensure an objective approach was adhered to. Furthermore, all screening (at title, abstract and full-text level) was performed by non-MSC staff. Metadata extraction will be performed by 2 non-MSC staff and 2 MSC staff. The majority (up to $75 \%$ ) of meta-data extraction will be performed by non-MSC staff to further reduce the risk of potential impartiality.

\section{Availability of data and materials \\ Not applicable.}

\section{Consent for publication}

Not applicable.

\section{Ethics approval and consent to participate}

Not applicable.

\section{Funding}

$\mathrm{AA}, \mathrm{FN}$ and $\mathrm{CL}$ are employees of the MSC and as such receive salaries from the organisation that is the focus of this systematic map protocol. In this way the MSC has contributed to the financing of this manuscript. However, the MSC is a registered non-profit organisation and charity.

\section{Publisher's Note}

Springer Nature remains neutral with regard to jurisdictional claims in published maps and institutional affiliations.

Received: 7 August 2018 Accepted: 3 December 2018

Published online: 10 December 2018 


\section{References}

1. MSC. Global Impacts Report 2016 London, England; 2016. https://www. msc.org/documents/environmental-benefits/global-impacts/msc-globa l-impacts-report-2016. Accessed 30 Nov 2018.

2. MSC. The MSC Standards. 2018. https://www.msc.org/standards-and-cert fication/the-msc-standards. Accessed 30 Nov 2018.

3. FAO. Code of conduct for responsible fisheries. Rome: Food and Agriculture Organization; 1995. p. 41.

4. Marine Stewardship Council. Marine Stewardship Council Certification Requirements Version 1.3. 2013. https://www.msc.org/docs/default-sourc e/default-document-library/for-business/program-documents/fisheriesprogram-documents/msc_certification_requirements_v1_3.pdf?sfvrs $\mathrm{n}=44528820$ 20. Accessed 30 Nov 2018.

5. Marine Stewardship Council. Harnessing market forces for positive environmental change: the MSC theory of change. 2011 p. 1-5. https://www. msc.org/docs/default-source/default-document-library/what-we-aredoing/msc-theory-of-change-2011.pdf?sfvrsn=2745eda8_18. Accessed 30 Nov 2018.

6. Bellchambers LM, Gaughan DJ, Wise BS, Jackson G, Fletcher WJ. Adopting Marine Stewardship Council certification of Western Australian fisheries at a jurisdictional level: the benefits and challenges. Fish Res. 2016;183:60916. https://www.scopus.com/inward/record.uri?eid=2-s2.0-8498993145 $1 \&$ doi $=10.1016 \% 2 F j . f i s h r e s .2016 .07 .014 \&$ partnerlD=40\&md5=9a705 6ebc4cda25d5f712dc1d69c85d8.

7. Adolf S, Bush SR, Vellema S. Reinserting state agency in global value chains: The case of MSC certified skipjack tuna. Fish Res. 2016;182:79-87. https://doi.org/10.1016/j.fishres.2015.11.020.

8. Bellchambers LM, Phillips BF, Pérez-Ramírez M. From certification to recertification the benefits and challenges of the Marine Stewardship Council (MSC): a case study using lobsters. Fish Res. 2015;182:88-97.

9. Gutiérrez NL, Valencia SR, Branch TA, Agnew DJ, Baum JK, Bianchi PL, et al. Eco-label conveys reliable information on fish stock health to seafood consumers. PLoS One. 2012;7(8). https://www.scopus.com/inward/recor d.uri?eid=2-s2.0-84865172648\&doi=10.1371\%2Fjournal.pone.00437 65\&partner $\mid D=40 \& m d 5=559 a 696679 c 97 a d 3 a 1 a 381 c 0025 c 0252$

10. Roheim CA, Asche F, Santos Jl. The elusive price premium for ecolabelled products: evidence from seafood in the UK market. J Agric Econ. 2011:62(3):655-68.

11. Stemle A, Uchida H, Roheim CA. Have dockside prices improved after MSC certification? Analysis of multiple fisheries. Fish Res. 2016;182:11623. https://doi.org/10.1016/j.fishres.2015.07.022.

12. Karavias M. Interactions between International Law and Private Fisheries Certification. Transnational Environmental Law. 2017. p. 1-20. https://www.scopus.com/inward/record.uri?eid=2-s2.0-8502676949 $5 \&$ doi $=10.1017 \% 2 F S 2047102517000139 \&$ partner $\mid \mathrm{D}=40 \& \mathrm{md} 5=25088$ 5419a116f6f52190c056d16563e. Accessed 30 Nov 2018.

13. Cooke SJ, Wesch S, Donaldson LA, Wilson ADM, Haddaway NR. A call for evidence-based conservation and management of fisheries and aquatic resources. Fisheries. 2017:42(3):143-9.

14. Araujo R, Bartsch I, Bekkby T, Erzini K, Sousa-Pinto I. What is the impact of kelp forest density and/or area on fisheries? Environ Evid. 2013;2:15.
15. Hughes KM, Kaiser MJ, Jennings S, McConnaughey RA, Pitcher R, Hilborn $\mathrm{R}$, et al. Investigating the effects of mobile bottom fishing on benthic biota: a systematic review protocol. Environ Evid. 2014;3(1):23.

16. Leisher C, Temsah G, Booker F, Day M, Samberg L, Prosnitz D, et al. Does the gender composition of forest and fishery management groups affect resource governance and conservation outcomes? A systematic map. Environ Evid. 2016:5(1):1-10.

17. Steering Committee of the State-of-Knowledge Assessment of Standards and Certification. Toward Sustainability: the roles and limitations of certification. Washington, DC; 2012. http://citeseerx.ist.psu.edu/viewdoc/downl oad?doi=10.1.1.117.3994\&amp;rep=rep1\&amp;type $=$ pdf\#page $=32$. Accessed 30 Nov 2018.

18. Petrokofsky G, Jennings S. The effectiveness of standards in driving adoption of sustainability practices: A State of Knowledge Review. Oxford, United Kingdom; 2018. https://www.standardsimpacts.org/sites/defau It/files/Full_report_The_effectiveness_of_standards_in_driving_adopt ion_of_sustainability_practices_FINAL.pdf. Accessed May 222018.

19. Komives K, Arton A, Baker E, Kennedy E, Longo C, Newsom D, et al. How has our understanding of the conservation impacts of voluntary sustainability standards changed since the 2012 publication of "Toward Sustainability: The Roles and Limitations of Certification?".

20. Petticrew M, Roberts H. Evidence, hierarchies, and typologies: horses for courses. J Epidemiol Community Health. 2003;57(7):527-9.

21. Haddaway N, Macura B, Whaley P, Pullin A. ROSES for systematic map protocols version 1.0. 2017. https://www.roses-reporting.com/roses-for-syste matic-review-protoco. Accessed 30 Nov 2018.

22. Pullin A, Frampton G, Livoreil B, Petrokofsky G. Guidelines and standards for evidence synthesis in environmental management. Collaboration for Environmental Evidence. 2018.

23. Haddaway NR, Kohl C, Da Rebelo Silva N, Schiemann J, Spök A, Stewart $R$, et al. A framework for stakeholder engagement during systematic reviews and maps in environmental management. Environ Evid. 2017;6(1):11.

24. Haddaway NR, Collins AM, Coughlin D, Kirk S. The role of google scholar in evidence reviews and its applicability to grey literature searching. PLoS ONE. 2015:10(9):1-17.

25. Cheng SH, Augustin C, Bethel A, Gill D, Anzaroot S, Brun J, et al. Using machine learning to advance synthesis and use of conservation and environmental evidence. Conserv Biol. 2018. https://doi.org/10.1111/ cobi.13117.

26. Altman D. Measuring Agreement. In: Altman D, editor. Practical statistics for medical research. London: Chapman and Hall; 1991.

27. Livoreil B, Glanville J, Haddaway NR, Bayliss H, Bethel A, De Lachapelle FF, et al. Systematic searching for environmental evidence using multiple tools and sources. Environ Evid. 2017;6(1):23.

28. Pullin S, Frampton G, Livoreil B, Petrokofsky G. Section 3. Planning a CEE evidence synthesis. In: Guidelines and standards for evidence synthesis in environmental management. Version 5. 2018. http://www.environmen talevidence.org/quidelines. Accessed 30 Nov 2018. 